\title{
EXCREÇÃO URINÁRIA DE FENOL EM TRABALHADORES DE INDÚSTRIAS DE CALÇADOS EXPOSTOS AO BENZENO *
}

\author{
Nilda Gallego Gandara de Fernícola ** \\ Celina Tamie Wakamatsu*** \\ René Mendes *** \\ Ester de Camargo Fonseca Moraes****
}

\begin{abstract}
RSPU-B/327
FERnícola, N. G. G. de et al. - Excreção urinária de fenol em trabalhadores de indústrias de calçados expostos ao benzeno. Rev. Saúde públ., S. Paulo, $10: 327-33,1976$.

RESUMo: Foi utilizada a excreção urinária de fenol como indicador biológico de exposição profissional ao benzeno, em uma amostra populacional de 79 trabalhadores de industrias de calçados no municipio de Franca, SP, Brasil $e$ em 65 pessoas que realizam trabalhos domésticos com colas, contendo benzeno, para industrias de calçados no município de São Paulo. Os valores médios encontrados, respectivamente 6,5 e $4,2 \mathrm{mg} / \mathrm{g}$ de creatinina, permitiram caracterizar diferentes graus de exposição. A primeira população rtrabalhado. res de indústrias de calçados de Franca), pôde ser considerada como um todo, pelo menos, no "período pré-patogênico" da intoxicação profissional pelo benzeno.
\end{abstract}

UNITERmos: Benzeno (indústria de calçados). Saúde ocupacional. Toxicologia industrial.

\section{I N TR O D U C A O}

A exposição profissional ao benzeno tem leves até às formas extremamente graves sido associada a efeitos tóxicos sobre o e mesmo fatais, como a anemia aplástica organismo, particularmente sobre o sistema hematopoiético, nas exposições a longo prazo. Estes efeitos traduzem-se por e a leucose ${ }^{16,19,28,38}$

Tais efeitos tóxicos sobre o homem já alterações hematológicas, desde as mais tura científica brasileira, sob a forma de

* Trabalho realizado na Fundação Centro Nacional de Segurança, Higiene e Medicina do Trabalho e Faculdade de Ciências Farmacêuticas da Universidade de São Paulo.

* Do Departamento de Análises Clínicas e Toxicológicas da Faculdade de Ciências Farmacêuticas da USP - Conjunto das Quimicas, Bloco 17 - Cidade Universitária, São Paulo, SP - Brasil; da Superintendência de Avaliação do Ruido e Qualidade do Ar, da Companhia Estadual de Tecnologia de Saneamento Básico e de Defesa do Meio Ambiente (CETESB) - Av. Prof. Frederico Hermann Júnior, 345 - São Paulo, SP Brasil.

*** Do Departamento de Medicina Preventiva da Escola Paulista de Medicina - Rua Botucatu, 720 - São Paulo, SP - Brasil; da Superintendência de Avaliação do Ruído e Qualidade do Ar da CETESB.

** Do Departamento de Análises Clínicas e Toxicológicas da Faculdade de Ciências Farmacêticas da USP. 
FERNfCOLA, N. G. G, de et al, - Excreção urinária de fenol em trabalhadores de Indústrias de calcados expostos ao benzeno. Rev. Saúde puibl., S. Paulo, 10:327-33, 1976.

estudos clínico-laboratoriais de intoxicaçôes graves ${ }^{9,26,27}$ ou, mais freqüentemente, em trabalhos didáticos ou de revisão $5,7,13 ; 25,29,30,31,33$.

Verifica-se pela literatura científica estrangeira que, entre as inúmeras possibilidades de exposição profissional ao benzeno, o trabalho em indústrias de calçados e outros artigos de couro, pode constituir-se em importante risco profissional, se os trabalhadores nele envolvidos manipularem colas cujos solventes contêm benzeno ${ }^{1,2}, 3,4,8,10,17,18,32$.

Em trabalho anterior a este * foi verificado que o teor de benzeno variou de 0,13 a $7,68 \mathrm{~g}$ por $100 \mathrm{~g}$, em amostras de colas para calçados adquiridas aleatoriamente em casas comerciais de duas áreas de importante significado na industrialização de calçados no Brasil: Franca, SP e município de São Paulo.

Estes resultados, aliados a uma grande concentração de indústrias de calçados nestas áreas, levaram os autores a iniciar um estudo epidemiológico, visando avaliar o grau de exposição a que estão submetidos trabalhadores ligados a este ramo de atividade industrial, bem como os possíveis efeitos sobre a saúde, atribuiveis à ação tóxica deste solvente.

Para conseguir o primeiro objetivo avaliar o grau de exposição ao benzeno - escolheu-se como indicador biológico a eliminação do fenol urinário. Este exame, tido como o melhor indicador para a avaliação da quantidade de benzeno biotransformado $11,15,22,85,36,37,89$, pode ser considerado de alta especificidade e sensibilidade.

Atualmente, a utilização de técnicas mais modernas, como a cromatografia de fase gasosa, em lugar das tradicionais técnicas colorimétricas ${ }^{6}, 12,20,24$, leva ainda a melhor termo a fidelidade desta avaliação.

- Dados inéditos

\section{MATERIAL E MÉtOdOS}

Foi recolhida a urina de uma micção, ao final da jornada de trabalho, de 79 (setenta e nove) trabalhadores de indústrias de calçados em Franca, SP e de 65 (sessenta e cinco) pessoas que realizam trabalhos domésticos de preparação da parte superior dos calçados, desde a colagem do couro até o pesponto, para as indústrias de calçados, no município de São Paulo. As amostras de urina foram conservados em geladeira, a $4^{\circ} \mathrm{C}$, até sua análise.

De cada amostra foram determinados os valores da creatinina e do fenol, este por cromatografia de fase gasosa, segundo Buchet et al. ${ }^{6}$.

\section{RESULTADOS}

O valor médio do fenol urinário das amostras de urina dos trabalhadores das indústrias de calçados em Franca, SP, foi $6,5 \mathrm{mg} / \mathrm{g}$ de creatinina, com $3,6 \mathrm{mg} / \mathrm{g}$ de creatinina de desvio padrão. 0 valor médio do fenol urinário de amostras de pessoas que realizam trabalhos domésticos de colagem de couros em São Paulo, foi de $4,2 \mathrm{mg} / \mathrm{g}$ de creatinina, com $3,0 \mathrm{mg} / \mathrm{g}$ de creatinina de desvio padrão. $\mathrm{Na} \mathrm{Fi-}$ gura 1 está apresentada a distribuição percentual dos trabalhadores de Franca e de São Paulo por classes de fenol urinário, bem como a distribuição dos não expostos. tomados como normais.

\section{D I S U S S O}

Embora a eliminação urinária de fenol seja o exame mais freqüentemente recomendado para avaliar o grau de exposição ao benzeno ${ }^{11,15,36,37,39}$, não existe muita harmonia entre os diferentes autores, quer sobre os valores da normalidade e o significado de valores mais elevados de excreção, quer sobre a própria unidade utilizada para expressar os resultados. A Tahela 1 reúne alguns valores referidos por diferentes autores. 
FERniColA, N. G. G. de et al. - Excreção urinária de fenol em trabaihadores de indústrias de calçados expostos ao benzeno. Rev. Saúde públ., S. Paulo, 10:327-33, 1976.

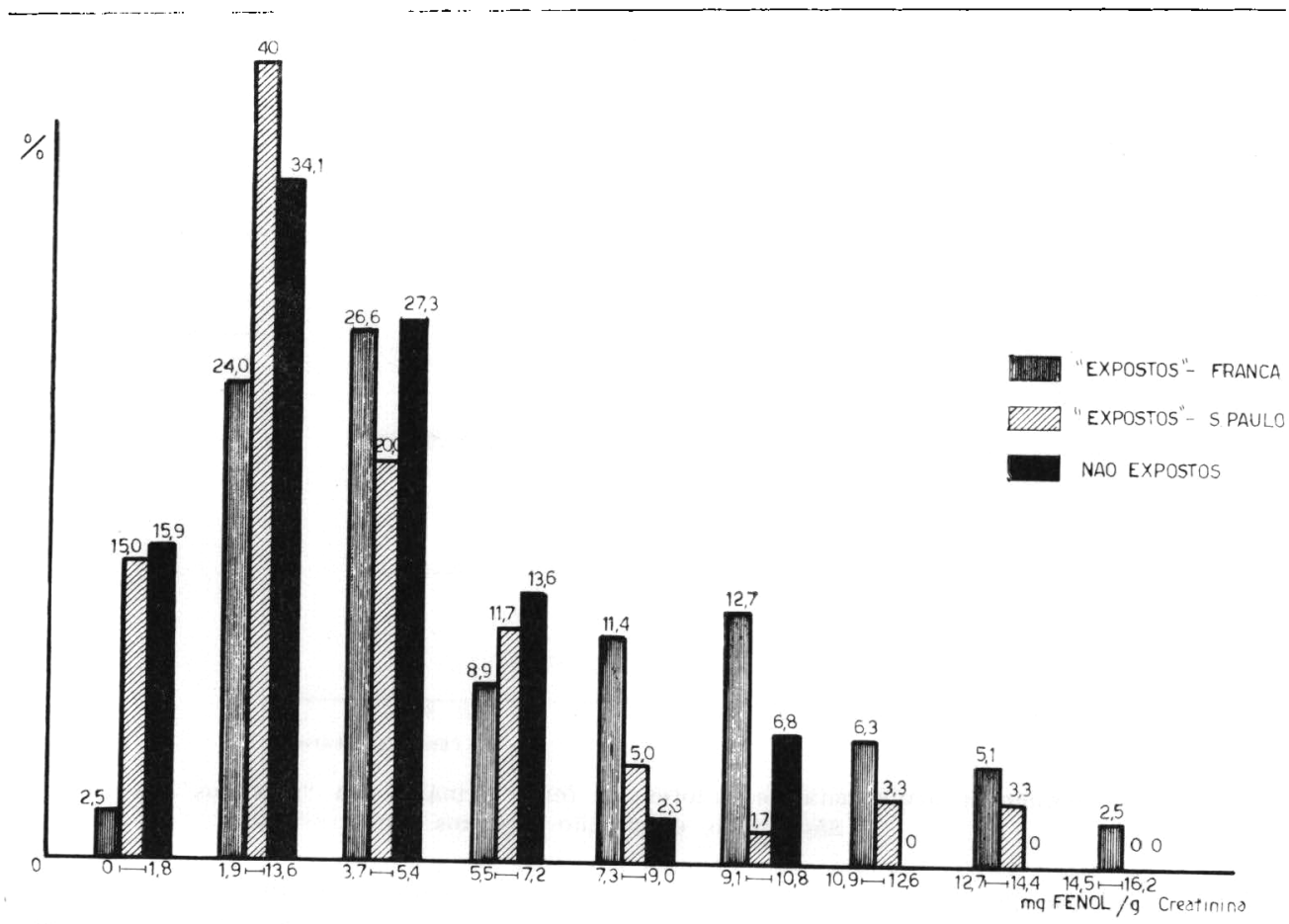

Fig. 1 - Distribuição percentual de valores de fenol urinário em "expostos" de Franca. São Paulo, e em não expostos.

TA B E A 1

Comparacão de valores de fenol urinário considerados como normais (por cromatografia de fase gasosa) referidos por diferentes autoles

\begin{tabular}{|c|c|}
\hline Autores & Valores de fenol urinário \\
\hline $\begin{array}{l}\text { Lebbe et } \\
\text { al. } 34\end{array}$ & $\begin{array}{ll}10,4 & \mathrm{mg} / 1200 \mathrm{ml} \text { (homens) } \\
11,3 \mathrm{mg} / 1200 \mathrm{ml} \text { (mulheres) }\end{array}$ \\
\hline Sherwood so & $1,8 \mathrm{mg} / 1 \quad(\mathrm{D}=1.017)$ \\
\hline Buchet et al." & $4,7 \mathrm{mg} / \mathrm{g}$ creatinina \\
\hline Duran et al. ${ }^{22}$ & 0,06 moles $/ g$ creatinina \\
\hline $\begin{array}{l}\text { Van Haaften } \\
\& \text { Sie }^{\text {rT }}\end{array}$ & $\mathrm{mg} / 1 \quad(\mathrm{D}=1.024)^{*}$ \\
\hline Hunter 21 & $0,41 \mathrm{mg} / \mathrm{h}$ \\
\hline $\begin{array}{l}\text { Fishbeck } \\
\text { et al. }{ }^{26}\end{array}$ & a $5 \mathrm{ppm}$ \\
\hline Sherwood ss & $\mathrm{mg} / 1 * *$ \\
\hline
\end{tabular}

* Valores acima de $10 \mathrm{mg} / \mathrm{l}$ indicam exposição e acima de $200 \mathrm{mg} / 1$ indicam exposições acima de $25 \mathrm{ppm}$ de benzeno no ar.

* Valores acima de $30 \mathrm{mg} / 1$ indicam provável exposição e acima de $100 \mathrm{mg} / 1$ indicam risco significativo
Frente a tão diferentes valores e critérios, tornou-se necessário definir valores ou faixas de normalidade efetivamente comparáveis, o que, aliás, já foi feito pelos autores, a partir da análise de uma população de indivíduos não expostos profissionalmente ao benzeno e que foi objeto de publicação anterior ${ }^{14}$. $O$ valor médio de normalidade encontrado pelos autores foi de $4,1 \mathrm{mg} / \mathrm{g}$ de creatinina, com $2,4 \mathrm{mg} / \mathrm{g}$ de creatinina como desvio padrão. A Figura 2 apresenta as curvas de frequêencia percentual acumulada para os dois grupos "expostos" - Franca, SP e São Paulo - e para o grupo "não expostos", tomado como "grupo controle".

A observação das Figuras 1 e 2 e dos valores médios apresentados permite admitir que os valores de fenol urinário dos trabalhadores das indústrias de calçados em Franca, são diferentes e maiores que os do "grupo controle". Esta diferença é estatisticamente significante, ao nível de 
FERNiCola, N. G. G. de et al. - Excreção urinárla de fenol em trabalnadores de indústrias de calcados expostos ao benzeno. Rev. Saude públ., S. Paulo, 10:327-33, 1976.

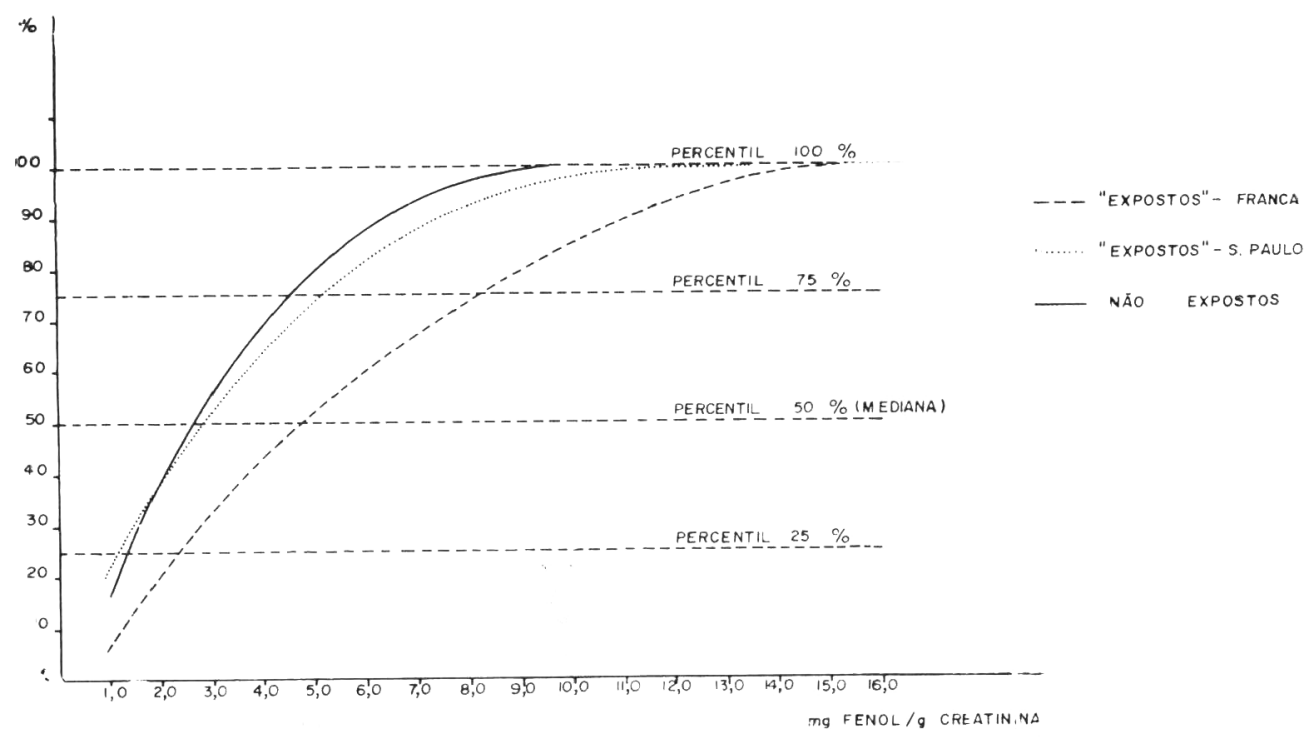

Fig. 2 - Freqüencia acumulada de valores de fenol urinário em "expostos" de Franca, São Paulo, e em não expostos.

$5.0 \%$. Embora pequena, esta diferença torna-se mais nítida através da apreciação das freqüências acumuladas (Fig. 2), principalmente dos percentis 50 (mediana), 75 e 100 .

O mesmo não pode ser dito para a população dos "expostos" de São Paulo, cujo valor médio da excreção urinária de fenol $(4,2 \mathrm{mg} / \mathrm{g}$ de creatinina) não difere, estatisticamente, do valor adotado como normal $(4,1 \mathrm{mg} / \mathrm{g}$ de creatinina). A curva de frequiência percentual acumulada está praticamente superposta à dos "não expostos".

A interpretação destes valores deve ser feita paralelamente à análise de características ocupacionais que, de fato, permitem distinguir entre $s i$, as duas populaçōes de "expostos".

Assim, a "exposição" dos trabalhadores de indústrias de calçados de Franca, fazse de modo praticamente contínuo, devido às características do trabalho em série, próprio das indústrias deste gênero, durante mais de oito horas por dia, cinco dias por semana. Além disto, o confinamento de vários trabalhadores, num mes- mo local, propicia a que todos se exponham, menos ou mais acentuadamente, mesmo que nem todos estejam continuamente manipulando as colas, em sua atividade individual.

Ao contrário, em São Paulo, na atividade escolhida, isto é, a realizada em trabalhos domésticos contratados pelas indústrias, existem fatores atenuantes da exposição. De fato, esta atividade não se faz continuamente no decorrer do dia, nem tão pouco diariamente, no decorrer da semana. Além disto, com raras exceções, em cada domicílio não trabalham mais do que uma ou duas pessoas, o que, naturalmente, diminui a presença de fontes de contaminação ambiental por benzeno e estas fontes podem apresentar quantidades variáveis do agente tóxico, uma vez que são fornecidas em pequenas quantidades pelos empresários.

Convém, no entanto, ressaltar que, a despeito destas características atenuantes do grau de exposição nas atividades domiciliares com colas contendo benzeno, tais atividades podem fazer com que outras pessoas mais suscetíveis aos efeitos tóxicos do benzeno - principalmente crianças 
FERNiCOLA, N. G. G. de et al. - Excreção urinária de fenol em trabalhadores de indústrias de calçados expostos ao benzeno. Rev. Saúde públ., S. Paujo, 10:327-33, 1976

- passem a se expor ao agente em questão.

Naturalmente, o estudo completar-se-ia com a avaliação ambiental, isto é, a determinação quantitativa de benzeno no ar. $\mathrm{Na}$ impossibilidade de realizar. correta e adequadamente esta determinação, o estudo da exposição ficou definido pelas três informações básicas caracterizadas: presença de benzeno nas colas, manipulação continua destas colas e aumento de fenol urinário. Esta tríade è suficiente para caracterizar, na linguagem de Leavell e Clark ${ }^{23}$, pelo menos o periodo pré-patogênico da história natural da intoxicação crônica pelo benzeno.

Obviamente, o estudo completa-se com a avaliaçāo dos possíveis efeitos sobre a saúde, atribuíveis à ação tóxica desse solvente. o que será objeto de futura publi‘ação.

\section{CONCLUSOES}

1. A determinação do fenol urinário (por cromatografia em fase gasosa) realizada em pessoas que manipulam colas contendo benzeno, permitiu caracterizar diferentes graus de exposição. compativeis com as característi- cas ocupacionais condicionantes de exposições de diferentes intensidades, previamente conhecidas.

2. A população que trabalha em indústrias de calçados. manipulando continuamente colas. pode ser considerada como um todo. "exposta" ao benzeno. situada. pelo menos, no "período pré-patogênico" da "história natural" da intoxicação crônica pelo benzeno. 10 valor médio de fenol urinário em 79 trahalhadores de indústrias de calçados de Franca. SP, foi $6.5 \mathrm{mg} / \mathrm{g}$ de creatinina e difere, significativamente. a nível de $5 \%$, do valor encontrado nos "não expostos").

3. A população que trabalha em atividades domésticas de colagem de couros para as indústrias de calçados. pode ser considerada, como um todo, potencialmente exposta ao henzeno. (O valor médio de fenol urinário em 65 pessoas que realizam esta atividade no município de São Paulo, foi de $4.2 \mathrm{mg} / \mathrm{g}$ de creatinina. não diferindo, a nível de $5 \%$, do valor médio encontrado nos "não expostos"

Fernicola, N. G. G. de et al. - TUnmary output of phenol in workmen exposed to benzene in the shoe industryl. Rev. Saúde puibl., S. Paulo, 10:327-33. 1976.

SUMMARY: The authors analised the urinary excretion of phenol of a groul of 79 shoemakers from Franca (State of $\mathrm{S}$. Paulo - Brazll and of 65 persons who make shoes at home for shoe factories in the city of Säo Panlo, in order to evaluate their occupational exposure to benzene present in the adhesives they use. The average values of $6.5 \mathrm{mg} / \mathrm{g}$ and $4.2 \mathrm{mg} / \mathrm{g}$ creat, respectively, lead the anthors to conclude that the two populations have different degress of erposure. The first population (woikers from Francal ought to be placea. at least in the prepathogenic period of chronic occupational poisoning.

UNITERMS: Benzene (shoe factories). Toxicology, industrial. Occupational health. 
FERNiCOLA, N. G. G. de et al. - Excreção urinária de fenol em trabalhadores de indús. trıas de calçados expostos ao benzeno. Rev. Saúde públ., S. Paulo, 10:327-33, 1976

\section{REFERENCIAS BIBLIOGRAFICAS}

1. AKSOY, M. et al. - Acute leukemia due to chronic exposure to benzene. Amer. J. Med., 52:160-5, 1972.

2. AKSOY, M, et al. - Details of blood changes in 32 patients with pancytopenia associated with long term exposure to benzene. Brit. J. industr. Med., 29:56-64, 1972.

3. AKSOY, MI. et al. - Haematological effects of chronic benzene poisoning in 217 workers. Brit. J. industr. Med., 28:296-302, 1971 .

4. AKSOY, M, et al. - Leukemia in shoeworkers exposed chronically to benzene. Blood, 44:37-41, 1974.

5. BENZOLISMO IBS Informativo, São Paulo, 5(52):3, 1972 .

6. BLCHET, J. P. et al. - An improved gas chromatographic method for the determination of phenol in urine. Europ. J. Toxicol., 5:27-30, 1972.

7. BLSTAMante, E. S. de - Benzolismo profissional. Med. Eng. Trab., 2: 31-9, 1956.

8. CALABUig ALBORCH, J. R. et al. Aplasias medulares benzolicas en la pequeña industria. Med. Segur. Trab., Madrid, 20(80):29-34, 1972.

9. CILlO, D M. - Aplasia medular ad. quirıda. São Paulo, 1966. [Tese Faculdade de Medicina LSP].

10. DI Bosco, M. M - Considerazioni su alcuni casi di leucosi da benzolo in operai dei calzaturifici. Lav. umano, $16: 105-21,1964$.

11. DOCTER, H J \& ZIELHUIS, R. L. Phenol excretion as a measure of benzene. Ann. occup. Hyg., 10:317-26, 1967.

12. DURAN, $M$ et al. - Gas chromatographic analysis of urinary volatile phenols in patients with gastrointes. tinal disorders and normals. Clin. chim. Acta, 45:341-7, 1973.

13. FARIA, G. S. - Benzolismo profissional. Impr. med., Rio de Janeiro, 21:7-12, 1945.

14. FERNICOLA. $\stackrel{\wedge}{G} G$ et al. - valor normal de fenol urinário. [Traba-
Iho apresentado no XIV Congresso Nacional de Prevenção de Acidentes do Trabalho, Rio de Janeiro, 1975].

15. FISHBECK, W. A. et al. - Elevated urinary phenol levels not related to benzene exposure Amer. industr. Hyg. Ass. J., 36:820-4, 1975.

16. GERARDE, H. W. - Toxicology and biochemistry of aromatic hydrocarbons. Amsterdam, Elsevier, 1960.

17. GIRARD, R. et al. - Les expositions benzéniques méconnues. Leur recherche systématique au cours des hémopathies graves. Arch. Mal. prof,, 29:723-6, 1968 .

18. GIRARD, $R$ et al. - Hydrocarbures benzéniques et hémopathies graves. Arch. Mal. prof., 31:625-35, 1970.

19. HANILTON, A. \& HARDY, H. L - I $t$ dustrial toxicology. $3^{\text {rd }}$ ed. Acton. Publishing Sciences Group, 1974.

20. HEISTAND, R. N. \& TODD, A. S. Automated determination of total phenol in urine. Amer. industr. Hyg. Ass. J., 33:378-81, 1972 .

21. HUNTER, C. G. - Solvents with reference to studies on the pharmacodinamies of benzene. Proc. roy. Soo. Med., 61:913-5, 1968.

22. HUNTER, C. G. \& BLAIR, D. - Benzene: pharmacokinetic studies in man. Ann. occup. Hyg, 15:193-9, 1972 .

23. LEAVELL, H. D. \& CLARK, E. G. Preventive medicine. $3^{\text {rd }}$ ed. New York, McGraw-Hill, 1965.

24. LEBBE, J. et al. - Recherche et dosage des monophénols urinaires par chromatographie en phase gazeuse Arch. Mal. prof., 27:565-9, 1966.

25. MENEzes, A. J. P. - Consideraçóes a respeito das alterações hematológlcas nas doenças profissionais. Impr. méd., Rio de Janeiro, 22:38-41. 1946.

26. MORRONE, L. C. \& ANDRADE, MI. Anemia aplástica pelo benzeno em uma Indústria de equipamentos plás- 
FERNicola, N. G. G. de et al. - Excreção urinárla de fenol em trabalhadores de incústrias de calsados expostos ao benzeno. Rev. Saúde públ., S. Paulo, 10:327-33, 1976

ticos - ocorrência de quatro casos fatais. In: CONGRESSO NACIONAL DE PREVENCEAO DE ACIDENTES DO TRABALHO, 13.0, São Paulo, 1974. Anais. São Paulo, 1974. p. 733-9.

27. Oliveira, H. C. P. de - Anemias aplásticas e agentes mielotóxicos. Rio de Janeiro, 1970. [Tese docência livre - Faculdade de Medicina - UFRJ].

28. ORGANIZACAO INTERNACIONAL DO TRABALHO - El benceno: sus utilizaciones, sus riesgos para la salud, su substitucion. Ginebra, 1968.

29. PARREIRAS, D. - Alteraçoes do sangue de origem profissional. Impr. méa., Rlo de Janelro, 26:58-63, 1950.

30. PARREIRAS, D. - Doencas profissionais do sangue e de seus órgãos de formação. Rev. bras. Med. puibl., 1: 31-7, 1945

31. PROTECAO contra os perigos do benzeno. CIPA J., 24(147):14-5, 1973.

32. REJSEK, $K$. \& REJSKOVA, M. - Long term observation of chronic benzene poisoning. Acta med. scand., 152: 71-8, 1955.
33. O RISCO do benzeno e a regulamentacão internacional. O Acidente, 6(3): 3 , 1971.

34. ROSSI, A. L. R. et al. - Dosagem de creatinina na urina. In: ROSSI, A. L. R. et al, - Práticas de bioquimica clinica, São Paulo, Ed. Universidade de Săo Paulo, 1973.

35. SHERWOOD, R. J. - Benzene: the interpretation of monitoring results. Ann. occup. Hyg., 15:409-21, 1972.

36. SHERWOOD, R. J. - Evaluation of exposure to benzene vapour during the loading of petrol. Brit. J. industr. Med., 29:65-9, 1972.

37. VAN HAAFTEN, A. B. \& SIE, S. T. The measurement of phenol in urine by gas chromatography as a check on benzene exposure. Amer. industr. Hyg. Ass. J., 26:52-8, 1965.

38. VIGLIANI, E. C. \& SAITA, G. - Benzene and leukemla. New Engl. J. Med., 2r1:872-6, 1964.

39. WALKLEY, J. E. et al. - The measurement of phenol in urine as an index of benzene exposure. Amer. industr. Hyg. Ass. J., 22:36-7, 1961.

Recebido para publicagão em 10/05/1976 Aprovado para publicacão em 14/06/1976 